\title{
Gravity with a Linear Action and Gravitational Singularities
}

\author{
George Savvidy* \\ Institute of Nuclear and Particle Physics \\ Demokritos National Research Centre \\ Athens, Greece \\ E-mail: savvidy@inp.demokritos.gr
}

\begin{abstract}
Motivated by quantum-mechanical considerations we earlier suggested an alternative action for discretised quantum gravity which measures the perimeter of the space-time and has a dimension of length. It is the so called perimeter action, since it is a "square root" of the area action in gravity and has a new constant of dimension one in front. The physical reason to introduce the perimeter/linear action was to suppress singular configurations "spikes" in the quantum-mechanical integral over geometries. Here we shall consider the continuous limit of the discretised perimeter action. We shall demonstrate that in the modified theory during the time evolution of a large massive star, when a star undergoes a collapse and develops an event horizon which confines the light, a smaller space-time region will be created behind the event horizon which is unreachable by test particles. These regions are located in the places where a standard theory of gravity has singularities. We are confronted here with a drastically new concept that during the time evolution of a massive star a space-time region is created which is excluded from the physical scene, being physically unreachable by test particles or observables. If this concept is accepted, then it seems plausible that the gravitational singularities are excluded from the modified theory.
\end{abstract}

Corfu Summer Institute 2017 "School and Workshops on Elementary Particle Physics and Gravity"

2-28 September 2017

Corfu, Greece

${ }^{*}$ Speaker. 


\section{Area Action versus Perimeter Action}

Unification of gravity with other fundamental forces within the superstring theory stimulated the interest to the theory of quantum gravity and to physics at Planck scale $[1,2,3,4,5]$. In particular, string theory predicts modification of the gravitational action at Planck scale with additional high derivative terms. This allows to ask fundamental questions concerning physics at Planck scale referring to these effective actions and, in particular, one can try to understand how they influence the gravitational singularities $[6,7,8,9,10,12,13,14,15,16,17,18,19,20,21,22,23,24]$.

It is appealing to extend this approach to different modifications of classical gravity which follow from string theory and also to develop an alternative approach which is based on new geometrical principles $[33,34,35]^{1}$. This approach to quantum gravity is based on the idea that the quantum mechanical amplitudes should be proportional to the "linear size" of the geometrical fluctuations of the space time manifold. This principle will allow to extend the notion of the Feynman integral over paths to an integral over space-time manifolds so that when a manifold collapses into a single world line the corresponding quantum-mechanical amplitude becomes proportional to the length of the world line. In other words, in this limit the gravitational action should reduce to the relativistic particle action which is equal to the length of the world line and measures it in $\mathrm{cm}$ [39].

The legitimate question to be asked is why to consider alternative geometrical principles? The reason is that when the action has a dimension larger than one, that is, the action has dimension $\mathrm{cm}^{d}$, where $d>1$, then the geometrical fluctuations of lower dimension will grow uncontrollably on a space-time manifold. This happens because the action is "blind" toward measuring the low dimensional fluctuations [36, 37, 38, 39]. Indeed, let us consider a discretised two-dimensional world sheet surface and a theory in which the action is equal to the area of the surfaces. The Feynman integral here is an integral over all vertices of the triangulated surface. The fluctuations will grow on a surface in the form of tine spikes, because spikes have zero area and will be created with a large amplitude of order one. The benefit of introducing a perimeter/linear action is that it suppresses singular configurations in the form of "spikes" in the quantum-mechanical integral over geometries, thus suppressing any fluctuation of lower dimensionality.

One can demonstrate this phenomenon on a beautiful example from Integral Geometry $[30,31,32]$. Let us consider a random triangle $A B C$ on a two-dimensional plane which is created by three randomly distributed vertices $\vec{x}_{1}, \vec{x}_{2}, \vec{x}_{3}$ [31]. The measure which is invariant under the isometries of the Euclidean plane is (see Fig.1)

$$
d \mu=d^{2} x_{1} d^{2} x_{2} d^{2} x_{3},
$$

and we shall consider the partition functions when the action is proportional to the area of the triangle $S$ or to its perimeter $L$. Using the triangle angles $\alpha_{1,2,3}$ one can represent the measure $d \mu$ in the form $d \mu=d^{2} x_{1} d \phi \rho_{2} \rho_{3} d \rho_{2} d \rho_{3} d \alpha_{1}$, where $d^{2} x_{2}=\rho_{2} d \rho_{2} d \phi_{2}, \quad d^{2} x_{3}=$ $\rho_{3} d \rho_{3} d \phi_{3}, \quad \phi_{3}-\phi_{2}=\alpha_{1}$ and $\phi_{2}=\phi$ and then express it in the following form [31]:

\footnotetext{
${ }^{1}$ See also the references [36, 37, 38, 39].
} 


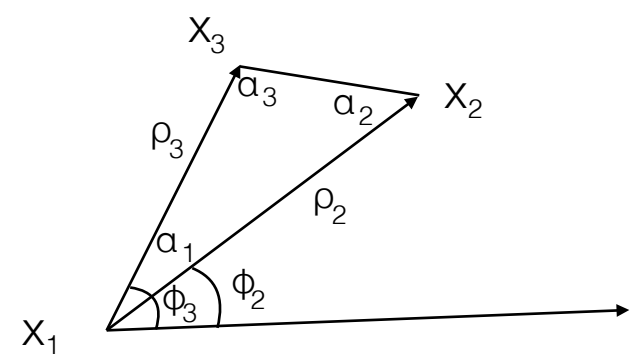

Figure 1: The measure which is invariant under isometries of the Euclidean plane is $d \mu=$ $d^{2} x_{1} d^{2} x_{2} d^{2} x_{3}$. It can be transformed into the form which is expressed in terms of geometrical characteristics of the triangle shape such as its area $S$ or perimeter $L$ and the angles $\alpha_{1}, \alpha_{2}$. The results are presented by formulas (1.1) and (1.2).

$$
d \mu_{S}=d^{2} x_{1} d \phi \quad S d S \frac{d \alpha_{1} d \alpha_{2}}{\sin \alpha_{1} \sin \alpha_{2} \sin \alpha_{3}}
$$

and as

$$
d \mu_{L}=d^{2} x_{1} d \phi \quad L^{3} d L \frac{\sin \alpha_{1} \sin \alpha_{2} \sin \alpha_{3}}{\left(\sin \alpha_{1}+\sin \alpha_{2}+\sin \alpha_{3}\right)^{4}} d \alpha_{1} d \alpha_{2}
$$

The part of the measure $d^{2} x_{1} d \phi$ factorises, it describes the translation and rotation of the triangle as a whole and therefore is irrelevant for our consideration. The rest of the measure allows to calculate the entropy, that is to answer the following question: how many of the randomly created triangles have the area $S$ or the perimeter $L$ ? For that one should integrate the measure in (1.1) and (1.2) over the independent parameters defining a geometrical shape of the triangles: $\alpha_{1}$ and $\alpha_{2}$ at fixed $S$ or $L$. The integral is logarithmically diverging in the area case (1.1) and is finite in the perimeter case (1.2). Thus the results are different: there are infinitely many triangles of fixed area, because the triangle can be infinitely long, in the form of a spike. In the case of the perimeter action (1.2) the integral is converging and is perfectly well defined. This example illustrates why the perimeter action has an advantage to define a geometrical theory in which the spiky configurations are suppressed.

In the case of a single triangle geometries it was clear what should be understood under its perimeter or of its linear size. The question is how to measure the perimeter/linear size of the high-dimensional manifolds and, in particular, a triangulated two-dimensional world sheet surface in terms of $\mathrm{cm}$, instead of the areas of its triangles. The invariant which characterises the linear size of the discretised two-dimensional surface can be constructed summing the lengths of its edges $l_{i j}$ multiplied by the deficit angle $\omega_{i j}=\left|\pi-\alpha_{i j}\right|$ on the corresponding edge $\langle i j\rangle[33,34,35,36,37,38,39]$ (see Fig.2)

$$
L=\sum_{<i j>\in \mathcal{M}_{2}} \lambda_{i j}\left|\pi-\alpha_{i j}\right|
$$

The action measures the surface in terms of $\mathrm{cm}$ and reduces to its length when the 


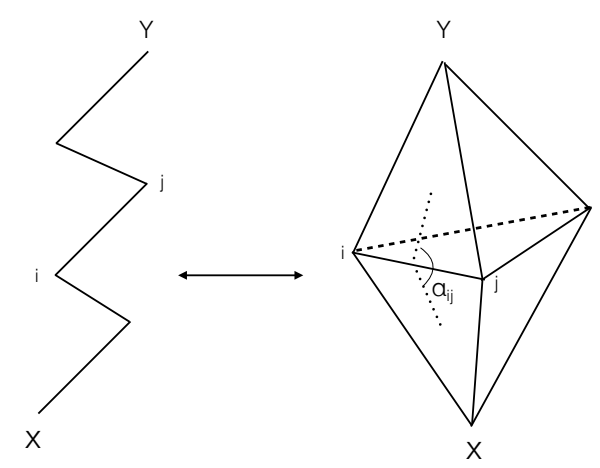

Figure 2: The discretised two-dimensional surface describing the propagation of a string from space-time point $X$ to $Y$. The action (1.3) allows to extend the notion of the Feynman integral over paths to an integral over space-time surfaces, so that when a two-dimensional surface degenerates into a single world line the quantum mechanical amplitude becomes proportional to the length of the world line : $L=\sum_{<i j>} \lambda_{i j} \longleftrightarrow L=\sum_{<i j>} \lambda_{i j}\left|\pi-\alpha_{i j}\right|$.

surface collapses into a single world line, as one can see on Fig.2. The deficit angle in the above formula plays an important role, because otherwise the action will be ill defined and unbounded, in particular, if one adds a flat edge with its dihedral angle $\alpha_{i j}=\pi$ it will not contribute into the action, only non-flat edges $\alpha_{i j} \neq \pi$ contribute into the sum over edges.

Let us now turn to a three-dimensional space-time manifolds representing a discretised quantum gravity. In that case the action was found by Regge and it has the form [41]:

$$
L=\sum_{<i j>\in \mathcal{M}_{3}} l_{i j} \omega_{i j}, \quad \omega_{i j}=\left(2 \pi-\alpha_{1}-\ldots-\alpha_{n}\right)_{i j}
$$

where $l_{i j}$ is the length of the edge $\langle i j\rangle$ and $\omega_{i j}$ is the deficit angle on the edge (see Fig.3). This action is the discretised version of the Hilbert-Einstein (HE) action, it has the dimension of length

$$
L=\int_{\mathcal{M}_{3}} R \sqrt{-g} d^{3} x
$$

and measures the linear size of the three-dimensional manifold in $\mathrm{cm}$.

Finally in four dimensions the HE action has dimension $\mathrm{cm}^{2}$ and measures the area of the universe. The dimension of the measure $\left[\sqrt{-g} d^{4} x\right]$ is $\mathrm{cm}^{4}$, the dimension of the scalar curvature $[R]$ is $1 / \mathrm{cm}^{2}$, thus the integral $\int R \sqrt{-g} d^{4} x$ has dimension $\mathrm{cm}^{2}$ and measures the area of the universe Fig.4. In the discretised representation the Regge action is the sum of areas of the triangles multiplied by the corresponding deficit angles [41]

$$
S=\sum_{<i j k>\in \mathcal{M}_{4}} \sigma_{i j k} \omega_{i j k}
$$

where $\sigma_{i j k}$ is the area of the triangle $\left\langle i j k>\right.$ and $\omega_{i j k}$ is the deficit angle on the triangle $<i j k>$. Here as well, there is a cone which appears in the normal section of the triangle $<i j k>$ and the deficit angle is equal to its curvature. The action represents the discretised 

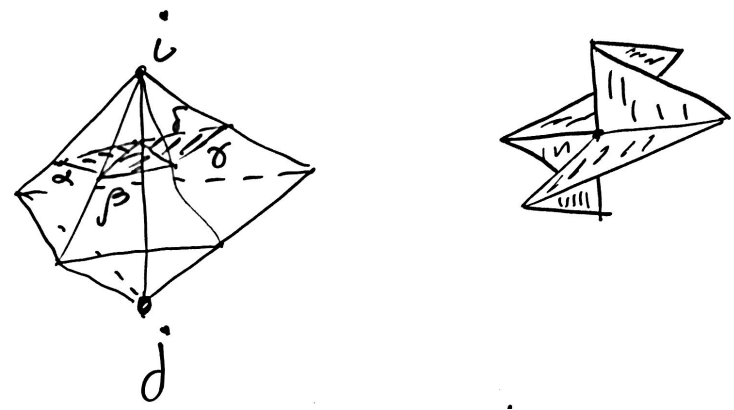

Figure 3: The example of discretised space-time manifold of the three-dimensional gravity. In three dimensions the Regge action (1.4) measures the linear size of the three-dimensional spacetime as it does its continuous counterpart (1.5). The Regge action (1.4) is a sum of lengths of all edges of the three-dimensional simplex multiplied by the deficit angles on cones which appear in the intersection of the edges by the normal planes. On the right hand side one can see an example of such a cone. The deficit angle is equal to the curvature of the cone $\omega_{i j}=(2 \pi-\alpha-\beta-\gamma-\delta)_{i j}$. If the cone is flat $(\alpha+\beta+\gamma+\delta)_{i j}=2 \pi$ then the deficit angle $\omega_{i j}$ is equal to zero and the edge does not contribute to the action.

version of the HE area action in four-dimensions:

$$
S=\int_{\mathcal{M}_{4}} R \sqrt{-g} d^{4} x
$$

We have been arguing above (1.1), (1.2) that the area action is unable to measure the one-dimensional singular configurations appearing in the form of tiny spikes and that the linear functional similar to the Feynman path integral action for the relativistic particles can represent a desired solution.

This raises a question: Is it possible to construct an action which measures the linear size of the four-dimensional manifold? It is not so difficult to construct an appropriate linear action starting from the Regge action (1.6) and taking instead of the triangle area $\sigma_{i j k}$ its perimeter $\lambda_{i j k}[33,34,35]$

$$
L=\sum_{<i j k>\in \mathcal{M}_{4}} \lambda_{i j k} \cdot \omega_{i j k}
$$

The linear character of the action (1.8) requires the existence of a new fundamental coupling constant $m_{P}$ of dimension $1 / \mathrm{cm}$. It is natural to call this action "perimeter/linear" or "gonihedric" because its definition contains the sum of products of the characteristic lengths and deficit angles. The action is well defined for discretised $4 D$ manifolds and can also be derived by postulating geometrical principles: the linearity and the continuity of the action functional.

Indeed, the discretised version of the linear action can be derived from: $\alpha$ ) the coincidence of the perimeter/linear action with the Feynman path integral in the cases when a manifold collapses to a single world line and $\beta$ ) the continuity of the transition amplitudes under the manifold deformations $[33,34,35]$. In accordance with $\alpha$ ) the quantum mechanical amplitude should be proportional to the length of the space-time manifold and 


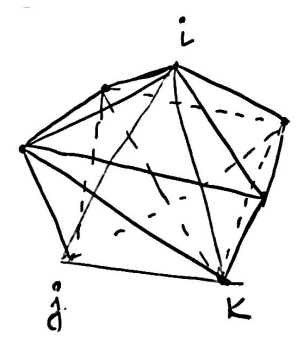

$$
\begin{aligned}
S\left(\mu_{4}\right) & =\sum_{\langle i j k\rangle} \sigma_{i j k} \cdot\left(2 \pi-\sum, s_{i, k}\right) \\
& =\int R^{(4)} d v_{4} \mathrm{~cm}^{2}
\end{aligned}
$$

Figure 4: The example of the simplicial space-time manifold of four-dimensional gravity. The Regge action (1.6) is a sum of all areas of the triangles multiplied by the deficit angles on a cone which appears in the intersection of the triangles by the normal planes. The perimeter/linear action (1.8) in four-dimensions is constructed by replacing the areas of the triangles $\sigma_{i j k}$ in (1.6) by the perimeters $\lambda_{i j k}$, as we were advocated in the text (1.1), (1.2) and demonstrated on Fig. 1.

therefore it must be proportional to the linear combination of the lengths of all edges of the discretised space-time manifold $\sum_{<i, j>} \lambda_{i j} \cdot \Theta_{i j}$, where $\lambda_{i j}$ is the length of the edge between two vertices $\langle i\rangle$ and $\langle j\rangle$, summation is over all edges $\langle i, j\rangle$ and $\Theta_{i j}$ is an unknown angular factor, which can be defined through the continuity principle $\beta$ ). The deficit angel $\Theta_{i j}$ should vanish in the cases when the triangulation around the edge $\langle i, j\rangle$ is flat, thus $\sum_{<i, j>} \lambda_{i j} \cdot \sum\left(2 \pi-\sum \beta_{i j k}\right)$, where $\beta_{i j k}$ are the angles on the cone which appear in the normal section of the edge $\langle i j\rangle$. Combining terms belonging to a given triangle $\langle i j k\rangle$ we shall get a sum $\lambda_{i j}+\lambda_{j k}+\lambda_{k i}=\lambda_{i j k}$ which is equal to the perimeter of the triangle $\langle i j k>$ and $\omega_{i j k}$ is the deficit angle on the triangle $\langle i j k>[33,34,35]$, thus recovering the action (1.8). Thus the principles of linearity and continuity allow to define the perimeter/linear action $L$ which can be considered as a "square root" of classical Regge area $S$ action (1.6) $[41,45]$.

Comparing the linear action $L$ in (1.8) and the Regge area action $S$ in (1.6) one should emphases that there is a deep analogy between these expressions and the earlier example considered in the beginning of this section where we were arguing that the perimeter action (1.2) has advantage compared with area action (1.1) since the integration over all geometries by the perimeter/linear action is well defined and nonsingular. Guided by this consideration that the perimeter/linear action has an advantage to suppress singular quantum-mechanical amplitudes, it is desirable to find a continuous counterpart of the perimeter/linear action (1.8). In case of the Regge action it was proven that its continuous limit reduces to the HE action (1.7) and our aim here is to address the same question in the case of the perimeter/linear action (1.8).

\section{Perimeter Action in Continuous Limit}

It is unknown to the author how to derive a continuous limit of the linear action (1.8) in a unique way. In this circumstance we shall try to construct a possible linear action for a smooth space-time universe by using the available geometrical invariants. Any expression which is quadratic in the curvature tensor and includes two derivatives could be a candidate 
for the linear action. The invariants we have chosen have the following form:

$$
I_{1}=-\frac{1}{180} R_{\mu \nu \lambda \rho ; \sigma} R^{\mu \nu \lambda \rho ; \sigma}, \quad I_{2}=+\frac{1}{36} R_{\mu \nu \lambda \rho} \square R^{\mu \nu \lambda \rho},
$$

and we shall consider a linear combination of the above expressions ${ }^{2}$ :

$$
L=-M c \int_{\mathcal{M}_{4}} \frac{3}{8 \pi}(1-\gamma) \sqrt{I_{1}+\gamma I_{2}} \sqrt{-g} d^{4} x,
$$

where we introduced the corresponding mass parameter $M$ and the dimensionless parameter $\gamma$. The dimension of the invariant $\left[\sqrt{I_{1}+\gamma I_{2}}\right]$ is $1 / \mathrm{cm}^{3}$, thus the invariant $L$ has the dimension of $\mathrm{cm}$ and measures the linear "size" of the universe. The expression (2.2) fulfils our basic physical requirement on the action that it should has the dimension of length and should reduce to the action of the relativistic particle in the situations when a manifold collapses to a one-dimensional curve

$$
L=-M c \int d s .
$$

Both expressions contain the geometrical invariants which are in general not positivedefinite under the square root. In the relativistic particle case (2.3) the expression under the root becomes negative for a particle moving with a velocity which exceeds the velocity of light. In that case the action develops an imaginary part and quantum-mechanical suppression of amplitudes prevents a particle from exceeding the velocity of light $[42,43,44]$. A similar mechanism was implemented in the Born-Infeld modification of electrodynamics with the aim to prevent the appearance of infinite electric fields [46, 48, 40]. It was found that there is a deep relation between the maximum field strength action and the fact that the D0-brane velocity is limited by the velocity of light [47]. The idea of a limiting curvature action was developed in the articles [16, 21, 22, 23] to prevent gravitational singularities.

One can expect that in the case of modified gravity with linear action $L$ in (2.2) there may appear space-time regions which are unreachable by the test particles if in that regions the expression under the root becomes negative. If these "locked" space-time regions happen to appear and if that space-time regions include singularities, then one can expect that the gravitational singularities are naturally excluded from the theory due to the fundamental principles of quantum mechanics. The question of consistency of the new action principle, if it is the right one, can only be decided by their physical consequences.

In the next sections we shall consider the black hole singularities and the physical effects which are induced by the inclusion of the linear action (2.2). As we shall see, the expression under the root becomes negative in the region which is smaller than the Schwarzschild radius $r_{g}$ and includes the singularities. For the observer which is far away from the horizon the linear action perturbation induces a small additional advance precession of the perihelion, but has a profound influence on the physics behind the horizon. We are confronted here with a drastically unusual concept that there may exist space-time regions which are excluded from the physical scene, being physically unreachable by test particles

\footnotetext{
${ }^{2}$ The general form of the action is presented in the Appendix.
} 
or observables. If one accepts this concept, then it seems plausible that the gravitational singularities are excluded from the modified theory. In this paper we have only taken the first steps to describe the phenomena which are caused by the additional linear term in the gravitational action proposed in [33, 34, 35].

The modified action which we shall consider is a sum

$$
S=-\frac{c^{3}}{16 \pi G} \int R \sqrt{-g} d^{4} x-M c \int \frac{3}{8 \pi}(1-\gamma) \sqrt{I_{1}+\gamma I_{2}} \sqrt{-g} d^{4} x
$$

where we introduced a dimensionless parameter $\gamma$ in order to consider a general linear combination of the invariants. The additional linear term has high derivatives of the metric and the equations of motion which follow from the variation of the action are much more complicated than in the standard gravity case, but because the linear action became relevant only in the situations when the metric is changing relatively fast one can consider as a first approximation the perturbation of the solutions of standard gravity generated by the additional linear term. It is obvious that in the space-time regions where the metric is varying slowly the modification of the standard gravity solutions should be negligible, at the same time the perturbations may became relevant in the space-time regions where curvature is large and the metric is changing relatively fast.

In this section we shall consider the perturbation of the Schwarzschild solution which is induced by the the additional linear term in the action and try to understand how it influences the black hole physics and the singularities. The Schwarzschild solution has the form

$$
d s^{2}=\left(1-\frac{r_{g}}{r}\right) c^{2} d t^{2}-\left(1-\frac{r_{g}}{r}\right)^{-1} d r^{2}-r^{2} d \Omega^{2},
$$

where $g_{00}=1-\frac{r_{g}}{r}, g_{11}=-\left(1-\frac{r_{g}}{r}\right)^{-1}, g_{22}=-r^{2}, g_{33}=-r^{2} \sin ^{2} \theta$, and

$$
r_{g}=\frac{2 G M}{c^{2}}, \quad \sqrt{-g}=r^{2} \sin \theta .
$$

The nontrivial quadratic curvature invariant in this case has the form

$$
I_{0}=\frac{1}{12} R_{\mu \nu \lambda \rho} R^{\mu \nu \lambda \rho}=\left(\frac{r_{g}}{r^{3}}\right)^{2}
$$

and shows that the singularity located at $r=0$ is actually a curvature singularity. The event horizon is located where the metric component $g_{r r}$ diverges, that is, at $r_{\text {horizon }}=r_{g}$. The expressions for the two curvature polynomials (2.1) of our interest are ${ }^{3}$ :

$$
I_{1}=\frac{r_{g}^{2}\left(r-r_{g}\right)}{r^{9}}, \quad I_{2}=\frac{r_{g}^{3}}{r^{9}},
$$

and on the Schwarzschild solution the action acquires additional term of the form

$$
L=-M c^{2} \int \frac{3}{2} \varepsilon \sqrt{1-\varepsilon \frac{r_{g}}{r}} \frac{r_{g}}{r^{2}} d r d t,
$$

\footnotetext{
${ }^{3}$ It should be stressed that all other invariant polynomials of the same dimensionality can be expressed in terms of $I_{1}$ and $I_{2}$, and they are given in Appendix.
} 
where

$$
\varepsilon=1-\gamma
$$

As one can see, the expression under the square root in (2.8) becomes negative at

$$
r<\varepsilon r_{g}, \quad 0<\varepsilon \leq 1
$$

and defines the region in which the action develops an imaginary part. Using the analogy with the relativistic particle action (2.3)

$$
L=-M c \int d s=-M c^{2} \int \sqrt{1-\frac{\vec{v}^{2}}{c^{2}}} d t .
$$

were the imaginary part is developing at the velocities which are larger than the velocity of light. In the last case this leads to the destructive superposition of quantum mechanical amplitudes outside the light-cone forbidding a particle to move with $v>c$. One can expect that in our case as well the development of the imaginary action in (2.8) will lead to region of the space-time which is unreachable by the test particles. The size of the region depends on the parameter $\varepsilon$ and is smaller than the gravitational radius $r_{g}$ if $\varepsilon$ is less than one (see Fig. 5).

This result seems to have profound consequences on the gravitational singularity at $r=$ 0. In a standard interpretation of the singularities, which appear in spherically symmetric gravitational collapse, the singularity at $r=0$ is hidden in the sense that no signal from it can reach infinity. The singularities are not visible for the outside observer, but hidden behind an event horizon. In that interpretation the singularities are still present in the theory. In the suggested scenario it seems possible to eliminate the singularities from the theory based on the fundamental principles of quantum mechanics. The singularities are excluded from the theory on the same level as the motion of particles with a velocity which exceeds the speed of light.

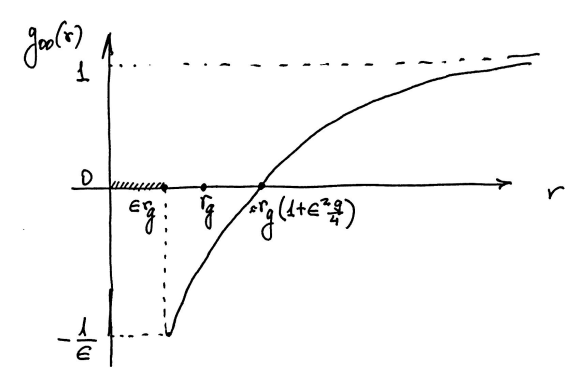

Figure 5: The graphic of the potential function $g_{00}=1-\frac{r_{g}}{r}-\left[1-\left(1-\varepsilon \frac{r_{g}}{r}\right)^{3 / 2}\right]^{2}$. At $r \rightarrow \infty$ the $g_{00} \rightarrow 1$ and at $r \rightarrow \varepsilon r_{g}$ the $g_{00} \rightarrow-\frac{1}{\varepsilon}$.

The quantum mechanical amplitude in terms of the path integral has the form

$$
\Psi=\int e^{\frac{i}{\hbar} S[g]} \mathcal{D} g_{\mu \nu}(x)
$$


where integration is over all diffeomorphism nonequivalent metrics. For the Schwarzschild massive object which is at rest we can find the expression for the action integrating (2.8)

$$
L=-M c^{2} \int_{\varepsilon r_{g}}^{\infty} \frac{3}{2} \varepsilon \sqrt{1-\varepsilon \frac{r_{g}}{r}} \frac{r_{g}}{r^{2}} d r d t=M c^{2} t
$$

and confirm that it is proportional to the length $t$ of the space-time trajectory, as it should be for the relativistic particle at rest, so that the corresponding amplitude can be written in the form

$$
\Psi \approx \exp \left(\frac{i}{\hbar} \sum_{n} M c^{2} t\right) .
$$

The perturbation (2.8) generates a contribution to the distance invariant $d s$ in (2.5) of the form

$$
\frac{3}{2} \int_{r}^{\infty} \varepsilon \sqrt{1-\varepsilon \frac{r_{g}}{r}} \frac{r_{g}}{r^{2}} d r=\left[1-\left(1-\varepsilon \frac{r_{g}}{r}\right)^{3 / 2}\right]
$$

and allows to calculate the correction to the purely temporal component of the metric tensor (2.5) caused by the additional term in the action

$$
g_{00}=1-\frac{r_{g}}{r}-\left[1-\left(1-\varepsilon \frac{r_{g}}{r}\right)^{3 / 2}\right]^{2} .
$$

Using the above expression of the metric one can analyse the influence of the perturbation on the physics at different regions of the space-time. The equation used to determine gravitational time dilation near a massive body is modified in this case and the proper time between events is defined now by the equation

$$
d \tau=\sqrt{g_{00}} d t=\sqrt{1-\frac{r_{g}}{r}-\left[1-\left(1-\varepsilon \frac{r_{g}}{r}\right)^{3 / 2}\right]^{2}} d t
$$

and therefore $d \tau \leq d t$, as in standard gravity. It follows from (2.16) that near the gravitational radius $r \approx r_{g}$ a purely temporal component of the metric tensor has the form

$$
g_{00} \approx 1-\frac{r_{g}}{r}-\varepsilon^{2} \frac{9}{4}\left(\frac{r_{g}}{r}\right)^{2}+\mathcal{O}\left(\varepsilon^{3}\right)
$$

confirming that the perturbation is small and the infinite red shift which appears in the standard case at $r=r_{g}$ now appears in its small vicinity

$$
r \approx r_{g}\left(1+\frac{9}{4} \varepsilon^{2}\right)+\mathcal{O}\left(\varepsilon^{4}\right) .
$$

To define the perturbation of the trajectories of the test particles outside of the massive body we shall study the behaviour of the solutions of the Hamilton-Jacobi equation for geodesics, which is modified by the perturbation of the metric:

$$
g^{\mu \nu} \frac{\partial A}{\partial x^{\mu}} \frac{\partial A}{\partial x^{\nu}}=g^{00}\left(\frac{\partial A}{c \partial t}\right)^{2}-\frac{1}{g^{00}}\left(\frac{\partial A}{\partial r}\right)^{2}-\frac{1}{r^{2}}\left(\frac{\partial A}{\partial \phi}\right)^{2}=m^{2} c^{2} .
$$

The solution has the form

$$
A=-E t+l \phi+A(r),
$$


where $E$ and $l$ are the energy and angular momentum of the test particle and

$$
A(r)=\int\left[\left(g^{00} \frac{E^{2}}{c^{2}}-m^{2} c^{2}-\frac{l^{2}}{r^{2}}\right) g^{00}\right]^{1 / 2} d r .
$$

In the non-relativistic limit $E=E^{\prime}+m c^{2}, E^{\prime} \ll m c^{2}$, and in terms of a new coordinate $r\left(r-r_{g}\right)=r^{\prime}$ we shall get

$$
A(r) \approx \int\left[\left(\frac{E^{\prime 2}}{c^{2}}+2 E^{\prime} m\right)+\frac{1}{r^{\prime}}\left(4 E^{\prime} m r_{g}+m^{2} c^{2} r_{g}\right)-\frac{1}{r^{\prime 2}}\left(l^{2}-\frac{3}{2} m^{2} c^{2} r_{g}^{2}\left(1+\frac{3}{2} \varepsilon^{2}\right)\right)\right]^{1 / 2} d r^{\prime} .
$$

The geodesic trajectories are defied by the equation $\phi+\partial A(r) / \partial l=$ Const and the advance precession of the perihelion $\delta \phi$ expressed in radians per revolution is given by the expression

$$
\delta \phi=\frac{3 \pi m^{2} c^{2} r_{g}^{2}}{2 l^{2}}\left(1+\frac{3}{2} \varepsilon^{2}\right)=\frac{6 \pi G M}{c^{2} a\left(1-e^{2}\right)}\left(1+\frac{3}{2} \varepsilon^{2}\right)
$$

where $a$ is the semi-major axis and $e$ is the orbital eccentricity. As one can see from the above result, the precession is advanced by the additional factor $1+\frac{3}{2} \varepsilon^{2}$. The upper bound on the value of $\varepsilon$ can be extracted from the observational data for the advanced precession of the Mercury perihelion, which is $42,98 \pm 0,04$ seconds of arc per century, thus

$$
\varepsilon \leq 0,16
$$

For the light propagation we shall take $m^{2}=0, E=\omega_{0}, l=\rho \omega_{0} / c$ in $(2.21)$ :

$$
A(r)=\frac{\omega_{0}}{c} \int \sqrt{\left(g^{00}-\frac{\rho^{2}}{r^{2}}\right) g^{00}} d r \approx \frac{\omega_{0}}{c} \int \sqrt{1+2 \frac{r_{g}}{r}-\frac{\rho^{2}}{r^{2}}} d r+\mathcal{O}\left(\varepsilon^{2} r_{g}^{2} / r^{2}\right) .
$$

The trajectory is defined by the equation $\phi+\partial A(r) / \partial \rho=$ Const and in the given approximation the deflection of light ray remains unchanged:

$$
\delta \phi=2 \frac{r_{g}}{\rho},
$$

where $\rho$ is the distance from the centre of gravity. The deflection angle is not influenced by the perturbation, which is of order $\mathcal{O}\left(\varepsilon^{2} r_{g}^{2} / \rho^{2}\right)$, and does not impose a sensible constraint on $\varepsilon$. In the next sections we shall consider perturbation of the Reissner-Nordström and the Kerr solutions.

\section{Reissner-Nordström Solution}

The Reissner-Nordström solution has the form

$$
d s^{2}=\left(1-\frac{r_{g}}{r}+\frac{r_{Q}^{2}}{r^{2}}\right) c^{2} d t^{2}-\left(1-\frac{r_{g}}{r}+\frac{r_{Q}^{2}}{r^{2}}\right)^{-1} d r^{2}-r^{2} d \Omega^{2}
$$

where

$$
r_{g}=\frac{2 G M}{c^{2}}, \quad r_{Q}^{2}=\frac{Q^{2} G}{c^{4}}, \quad \sqrt{-g}=r^{2} \sin \theta
$$


The nontrivial quadratic curvature invariant is

$$
I_{0}=\frac{1}{12} R_{\mu \nu \lambda \rho} R^{\mu \nu \lambda \rho}=\frac{3 r^{2} r_{g}^{2}-12 r r_{g} r_{Q}^{2}+14 r_{Q}^{4}}{3 r^{8}},
$$

and it shows that the singularity is located at $r=0$. The event horizon and internal Cauchy horizon are located where the metric component $g_{r r}$ diverges:

$$
r_{ \pm}=\frac{1}{2}\left(r_{g} \pm \sqrt{r_{g}^{2}-4 r_{Q}^{2}}\right) .
$$

The solutions with $r_{Q}>r_{g} / 2$ represent a naked singularity. As we shall see below, at these charges $r_{Q}$ the linear action develops a complex value and prevents the appearance of the naked singularities.

The expression for the two curvature polynomials of our interest are:

$$
\begin{aligned}
I_{1} & =\frac{\left(r^{2}-r r_{g}+r_{Q}^{2}\right)\left(45 r^{2} r_{g}^{2}-216 r r_{g} r_{Q}^{2}+304 r_{Q}^{4}\right)}{45 r^{12}}, \\
I_{2} & =\frac{9 r^{3} r_{g}^{3}+36 r^{3} r_{g} r_{Q}^{2}-96 r^{2} r_{g}^{2} r_{Q}^{2}-88 r^{2} r_{Q}^{4}+264 r r_{g} r_{Q}^{4}-200 r_{Q}^{6}}{9 r^{12}} .
\end{aligned}
$$

It is convenient to introduce the dimensionless quantities:

$$
\hat{r}=\frac{r}{r_{g}}, \quad \hat{r_{Q}}=\frac{r_{Q}}{r_{g}},
$$

and express the linear action on the Reissner-Nordström solution in the following form:

$$
L=-M c^{2} \int \frac{3}{2} \varepsilon \sqrt{\left(1-\frac{b}{\hat{r}}-\frac{c}{\hat{r}^{2}}+\frac{f}{\hat{r}^{3}}-\frac{e}{\hat{r}^{4}}\right)} \frac{1}{\hat{r}^{2}} d \hat{r} d t
$$

where

$$
\begin{gathered}
b=\varepsilon+\hat{r}_{Q}^{2} \frac{4(1+5 \varepsilon)}{5}, \quad c=\hat{r}_{Q}^{2} \frac{(219-480 \varepsilon)}{45}+\hat{r}_{Q}^{4} \frac{8(17-55 \varepsilon)}{45}, \\
f=\hat{r}_{Q}^{4} \frac{8(20-33 \varepsilon)}{9}, \quad e=\hat{r}_{Q}^{6} \frac{8(87-125 \varepsilon)}{45} .
\end{gathered}
$$

If the charge of the black hole is equal to zero, $\hat{r}_{Q}=0$, then the action (3.6) reduces to the expression (2.8) on the Schwarzschild solution. For the extremal black hole of the change $r_{Q}=r_{g} / 2$ the fourth order polynomial under the root in (3.6) is positive for $r>r_{g} / 2$, is equal to zero at $r=r_{g} / 2$ and is negative for $r<r_{g} / 2$. The region which is "locked" for the test particles in this case is defined by $r=r_{g} / 2$ and prevents the appearance of the naked singularities.

It is helpful to represent the polynomial under the square root in (3.6) in the form

$$
\left(1-\frac{b}{\hat{r}}-\frac{c}{\hat{r}^{2}}+\frac{f}{\hat{r}^{3}}-\frac{e}{\hat{r}^{4}}\right)=\left(1-\frac{\hat{r}_{1}}{\hat{r}}\right) \ldots\left(1-\frac{\hat{r}_{4}}{\hat{r}}\right),
$$

where $\hat{r}_{i}, i=1, \ldots, 4$ are the roots of the fourth order polynomial. The largest positive real valued root at which the polynomial turns out to be negative is defined as $\hat{r}_{4}$. Near that radius one can approximate the polynomial as

$$
\left(1-\frac{\hat{r}_{1}}{\hat{r}_{4}}\right)\left(1-\frac{\hat{r}_{2}}{\hat{r}_{4}}\right)\left(1-\frac{\hat{r}_{3}}{\hat{r}_{4}}\right)\left(1-\frac{\hat{r}_{4}}{\hat{r}}\right)=\Gamma\left(\hat{r}_{1}, \ldots, \hat{r}_{4}\right)\left(1-\frac{\hat{r}_{4}}{\hat{r}}\right) .
$$


Thus the action will take the form

$$
S \approx-M c^{2} \Gamma \int \frac{3}{2} \varepsilon \sqrt{\left(1-\frac{\hat{r}_{4}}{\hat{r}}\right)} \frac{1}{\hat{r}^{2}} d \hat{r} d t
$$

and it has a form similar to the case we had in the Schwarzschild black hole (2.8). As one can see, the expression under the square root in (3.9) becomes negative at

$$
r<r_{4}\left(r_{Q}, \varepsilon\right)
$$

and defines the region which is unreachable by the test particles.

The Table 1 presents the values of the radius $\hat{r}_{4}$ at which the polynomial under the root changes its sign from positive to negative and the action becomes complex as a function of the charge $\hat{r}_{Q}$ and the parameter $\varepsilon$. As it follows from the Table 1 for the extremal black hole, $\hat{r}_{Q}=1 / 2$, the "locked" region has the radius $\hat{r}_{4}=1 / 2$ and increases with the charge $\hat{r}_{Q}>1 / 2$, preventing the appearance of naked singularities. At $\hat{r}_{Q}=1$ the locked region has the radius $\hat{r}_{4} \approx 2.14$. For $\hat{r}_{Q}<1 / 2$ the locked region is smaller than horizon $\hat{r}_{4}<1 / 2$.

\begin{tabular}{lcc}
\hline $\begin{array}{l}\text { Charge } \\
\hat{r}_{Q}\end{array}$ & $\begin{array}{c}\text { Parameter } \\
\varepsilon\end{array}$ & $\begin{array}{c}\text { Maximal real solution } \\
\hat{r}_{4}\end{array}$ \\
\hline 1 & 0.1 & 2.14 \\
$1 / 2$ & 0.1 & 0.50 \\
$1 / 4$ & 0.1 & 0.47 \\
$1 / 8$ & 0.1 & 0.29 \\
$1 / 16$ & 0.1 & 0.18 \\
\hline
\end{tabular}

Table 1: The table of the solutions $\hat{r}_{4}$ at which the four order polynomial under the root function in (3.6) becomes negative. The value of $\hat{r}_{4}$ is measured in $r_{g}$ units (3.5). For the extremal black hole $\hat{r}_{Q}=1 / 2$ the "locked" region has the radius $\hat{r}_{4}=1 / 2$ and increases with the black hole charge $\hat{r}_{Q}>1 / 2$, thus preventing the appearance of naked singularities. For $\hat{r}_{Q}<1 / 2$ the locked region is smaller than horizon $\hat{r}_{4}<1 / 2$, that is $r_{4}<r_{g} / 2$.

\section{Kerr Solution}

Let us also consider the Kerr metric

$d s^{2}=\left(1-\frac{r_{g} r}{\rho^{2}}\right) c^{2} d t^{2}-\frac{\rho^{2}}{r^{2}-r_{g} r+a^{2}} d r^{2}-\rho^{2} d \theta^{2}-\left(r^{2}+a^{2}+\frac{r_{g} r a^{2}}{\rho^{2}} \sin ^{2} \theta\right) \sin ^{2} \theta d \phi^{2}+2 \frac{r_{g} r a}{\rho^{2}} \sin ^{2} \theta d \phi c d t$,

where

$$
\begin{gathered}
g_{00}=1-\frac{r_{g} r}{\rho^{2}}, g_{11}=-\frac{\rho^{2}}{r^{2}-r_{g} r+a^{2}}, g_{22}=-\rho^{2}, g_{33}=-\left(r^{2}+a^{2}+\frac{r_{g} r a^{2}}{\rho^{2}} \sin ^{2} \theta\right) \sin ^{2} \theta, \\
g_{03}=g_{30}=\frac{r_{g} r a}{\rho^{2}} \sin ^{2} \theta
\end{gathered}
$$

and

$$
a=\frac{J}{M c}, \quad r_{g}=\frac{2 G M}{c^{2}}, \quad \rho^{2}=r^{2}+a^{2} \cos ^{2} \theta, \quad \sqrt{-g}=\rho^{2} \sin \theta
$$


The nontrivial quadratic curvature invariant is

$$
I_{0}=\frac{1}{12} R_{\mu \nu \lambda \rho} R^{\mu \nu \lambda \rho}=\frac{r_{g}^{2}\left(r^{2}-a^{2} \cos ^{2} \theta\right)\left[\left(r^{2}+a^{2} \cos ^{2} \theta\right)^{2}-16 a^{2} r^{2} \cos ^{2} \theta\right]}{\left(r^{2}+a^{2} \cos ^{2} \theta\right)^{6}}
$$

and it shows that the singularity located at $r=0, \theta=\pi / 2$ is a curvature singularity. The event horizon is defined by the largest root of the equation $r^{2}-r_{g} r+a^{2}=0$ where the metric component $g_{11}$ diverges:

$$
r_{\text {horizon }}=\frac{1}{2}\left(r_{g}+\sqrt{r_{g}^{2}-4 a^{2}}\right) .
$$

For $a>r_{g} / 2$ there are no real valued solutions and there is no event horizon. With no event horizons to hide it from the rest of the universe, the black hole ceases to be a black hole and will instead be a naked singularity. The outer ergosurface is defined by the equation where the purely temporal component $g_{00}$ of the metric changes the sign from positive to negative:

$$
r_{\text {ergosur }}=\frac{1}{2}\left(r_{g}+\sqrt{r_{g}^{2}-4 a^{2} \cos ^{2} \theta}\right) .
$$

These two critical surfaces are tangent to each other at poles $\theta=0, \pi$ and they exist only when $a<r_{g} / 2$. The space between these two surfaces defines the ergosphere. At maximum value of the angular momentum $a=r_{g} / 2$ these surfaces are defined by the equations

$$
r_{\text {horizon }}=\frac{r_{g}}{2}, \quad r_{\text {ergosur }}=\frac{r_{g}}{2}(1+\sin \theta) .
$$

Let us now consider the expressions for the curvature polynomials $I_{1}$ and $I_{2}$ in the case of Kerr solution

$$
\begin{aligned}
& I_{1}=\frac{r_{g}^{2}\left(r^{2}-r_{g} r+a^{2} \cos ^{2} \theta\right)\left[r^{8}-28 a^{2} r^{6} \cos ^{2} \theta+70 a^{4} r^{4} \cos ^{4} \theta-28 a^{6} r^{2} \cos ^{6} \theta+a^{8} \cos ^{8} \theta\right]}{\left(r^{2}+a^{2} \cos ^{2} \theta\right)^{9}} \\
& I_{2}=\frac{\left.r r_{g}^{3}\left(r^{2}-3 a^{2} \cos ^{2} \theta\right)\left(r^{6}-33 a^{2} r^{4} \cos ^{2} \theta+27 a^{4} r^{2} \cos ^{4} \theta-3 a^{6} \cos ^{6} \theta\right)\right)}{\left(r^{2}+a^{2} \cos ^{2} \theta\right)^{9}}
\end{aligned}
$$

It is convenient to introduce the dimensionless quantities

$$
\hat{r}=\frac{r}{r_{g}}, \quad \hat{a}=\frac{a}{r_{g}}, \quad \sigma^{2}=\hat{a}^{2} \cos ^{2} \theta
$$

so that the linear action will takes the form

$$
S=-M c^{2} \int \frac{3}{2} \varepsilon \sqrt{f(\hat{r}, \hat{a}, \varepsilon, \theta)} \frac{1}{\hat{r}^{2}} d \hat{r} d t
$$

where

$$
\begin{aligned}
& f(\hat{r}, \hat{a}, \varepsilon, \theta)=\left(1-\frac{\varepsilon}{\hat{r}}-\frac{27 \sigma^{2}}{\hat{r}^{2}}-\frac{8 \sigma^{2}}{\hat{r}^{3}}+\frac{36 \sigma^{2} \varepsilon}{\hat{r}^{3}}+\frac{42 \sigma^{4}}{\hat{r}^{4}}+\frac{56 \sigma^{4}}{\hat{r}^{5}}-\frac{126 \sigma^{4} \varepsilon}{\hat{r}^{5}}+\right. \\
& \left.+\frac{42 \sigma^{6}}{\hat{r}^{6}}-\frac{56 \sigma^{6}}{\hat{r}^{7}}+\frac{84 \sigma^{6} \varepsilon}{\hat{r}^{7}}-\frac{27 \sigma^{8}}{\hat{r}^{8}}+\frac{8 \sigma^{8}}{\hat{r}^{9}}-\frac{9 \sigma^{8} \varepsilon}{\hat{r}^{9}}+\frac{\sigma^{10}}{\hat{r}^{10}}\right)\left(1+\frac{\sigma^{2}}{\hat{r}^{2}}\right)^{-9} \cdot
\end{aligned}
$$

The region which is locked for the test particles is defined by the largest real positive root of the polynomial (4.10) at which the polynomial turns out to be negative. It is denoted as 
$\hat{r}_{10}=\hat{r}_{10}(\hat{a}, \theta, \varepsilon)$. Let us consider the situation with maximal angular momentum $a=r_{g} / 2$ as in (4.6). The roots $\hat{r}_{10}(1 / 2, \theta, \varepsilon$,$) can be found numerically for different parameters of$ the Kerr solutions. In the maximal angular momentum case some of the values are:

$$
\begin{aligned}
& a=r_{g} / 2, \quad \theta=0, \pi \quad r_{\text {horizon }}=r_{g} / 2, \quad r_{\text {ergosur }}=r_{g} / 2, \quad r_{10}=2.6 r_{g} \\
& a=r_{g} / 2, \quad \theta=\pi / 2, \quad r_{\text {horizon }}=r_{g} / 2, \quad r_{\text {ergosur }}=r_{g}, \quad r_{10}=r_{g} / 2 .
\end{aligned}
$$

Thus the singularity is unreachable by the test particles. In the case of smaller angular momentum the locked region shrinks and is inside the event horizon.

In the above sections we were considered the perturbation of the exact solutions of the classical gravity by the linear action which suppresses the singular fluctuations. It is a difficult task to find the exact solutions of the equations which follow from the action (2.2) and (2.4) and we were unable to find exact solutions of these equations, but in the first approximation consider above the results are pointing out into the existence of the space-time regions surrounding the singularity which are inaccessible by the test particles.

In conclusion I would like to thank Jan Ambjorn for invitation and kind hospitality in the Niels Bohr Institute, where part of the work was done. I would like to thank Alex Kehagias for references and Kyriakos Papadodimas for useful remarks. The author acknowledges support by the ERC-Advance Grant 291092, "Exploring the Quantum Universe" (EQU) and COST Action MP1405. This lecture was presented in the Corfu Summer Institute 2017 "School and Workshops on Elementary Particle Physics and Gravity", 2-28 September 2017, Corfu, Greece and COST Action MP1405 "Quantum Structure of Spacetime" III. Annual Workshop: Quantum Spacetime '18, 19 - 23 February 2018, Sofia, Bulgaria.

\section{Appendix}

The general form of the linear action has the form:

$$
S_{L}=-m_{P} c \int \frac{3}{8 \pi} \sqrt{\sum_{1}^{3} \eta_{i} K_{i}+\sum_{1}^{4} \chi_{i} J_{i}+\sum_{1}^{9} \gamma_{i} I_{i}} \sqrt{-g} d^{4} x
$$

where the curvature invariants have the form

$$
\begin{aligned}
& I_{0}=\frac{1}{12} R_{\mu \nu \lambda \rho} R^{\mu \nu \lambda \rho}, \\
& I_{1}=-\frac{1}{180} R_{\mu \nu \lambda \rho ; \sigma} R^{\mu \nu \lambda \rho ; \sigma}, \\
& I_{2}=+\frac{1}{36} R_{\mu \nu \lambda \rho} \square R^{\mu \nu \lambda \rho}, \\
& I_{3}=-\frac{1}{72} \square\left(R_{\mu \nu \lambda \rho} R^{\mu \nu \lambda \rho}\right)=5 I_{1}-I_{2}, \\
& I_{4}=-\frac{1}{90} R_{\mu \nu \lambda \rho ; \alpha} R^{\alpha \nu \lambda \rho ; \mu}=I_{1}, \\
& I_{5}=-\frac{1}{18}\left(R_{\nu \lambda \lambda \rho}^{\alpha \nu} R_{\nu \lambda \rho ; \mu ; \alpha}^{\mu}=5 I_{1}-I_{2},\right. \\
& I_{6}=-\frac{1}{18}\left(R^{\alpha \nu \lambda \rho} R_{\nu \lambda \rho}^{\mu}\right)_{; \alpha ; \mu}=5 I_{1}-I_{2},
\end{aligned}
$$




$$
\begin{aligned}
& I_{7}=\frac{1}{18} R^{\alpha \nu \lambda \rho} R_{\nu \lambda \rho ; \alpha ; \mu}^{\mu}=I_{2}, \\
& I_{8}=R^{\mu}{ }_{\nu \lambda \rho ; \mu} R_{; \nu \lambda \rho}^{\sigma \nu,}, \\
& I_{9}=R^{\alpha \nu \lambda \rho} R_{\nu \lambda \rho ; \mu ; \alpha}^{\mu}, \\
& J_{0}=R_{\mu \nu} R^{\mu \nu}, \quad J_{1}=R_{\mu \nu ; \lambda} R^{\mu \nu ; \lambda}, J_{2}=R^{\mu \nu} \square R_{\mu \nu}, J_{3}=\square\left(R^{\mu \nu} R_{\mu \nu}\right), \quad J_{4}=R_{\mu \sigma}{ }^{; \mu} R_{; \nu}^{\nu \sigma} \\
& K_{0}=R^{2}, \quad K_{1}=R_{; \mu} R^{; \mu}, \quad K_{2}=R \square R, \quad K_{3}=\square R^{2} .
\end{aligned}
$$

The $\eta_{i}, \chi_{i}$ and $\gamma_{i}$ are free parameters. Some of the invariants can be expressed through others using Bianchi identities.

\section{References}

[1] A. Sakharov, Vacuum quantum fluctuations in curved space and the theory of gravitation, Dokl. Akad. Nauk SSSR 177 (1967) 70

[2] H.A. Buchdahl, Non-Linear Lagrangians and Cosmological Theory, Monthly Notices Roy. Astron. Soc. 150 (1970) 1 .

[3] A. A. Starobinsky, A New Type of Isotropic Cosmological Models Without Singularity, Phys. Lett. 91B (1980) 99. doi:10.1016/0370-2693(80)90670-X

[4] S. L. Adler, Einstein Gravity as a Symmetry Breaking Effect in Quantum Field Theory, Rev. Mod. Phys. 54 (1982) 729. doi:10.1103/RevModPhys.54.729

[5] M. Gasperini and G. Veneziano, Pre - big bang in string cosmology. , Astropart. Phys. 1 (1993) 317. doi:10.1016/0927-6505(93)90017-8.

[6] R. Penrose, Gravitational collapse and space-time singularities, Phys. Rev. Lett. 14 (1965) 57. doi:10.1103/PhysRevLett.14.57

[7] D.Christodoulou, Examples of naked singularity formation in the gravitational collapse of a scalar field. Ann. Math. 140 (1994) 607-653. doi:10.2307/2118619.

[8] S. Hawking, Occurrence of Singularities in Open Universes., Phys. Rev. Lett. 15 (1965) 689.

[9] S. Hawking, The Occurrence of Singularities in Cosmology., Proc. R. Soc. London A294 (1966) 511-521.

[10] S. Hawking, The Occurrence of Singularities in Cosmology. II. , Proc. R. Soc. London $\mathbf{A 2 9 5}$ (1966) 490-493.

[11] S. Hawking, The Occurrence of Singularities in Cosmology. III. Causality and Singularities., Proc. R. Soc. London A300 (1967) 187-201.

[12] S. Hawking and R. Penrose,, The singularities of gravitational collapse and cosmology., Proc. R. Soc. London A314 (1970) 529.

[13] H. P. Robertson, Relativistic Cosmology., Rev. Mod. Phys. 5 (1935) 62.

[14] A. Raychaudhure, Relativistic Cosmology I., Phys. Rev. 98 (1955) 1123.

[15] A. Komar, Necessity of Singularities in the Solution of the Field Equations of General Relativity., Phys. Rev. 104 (1956) 544.

[16] M. A. Markov, Limiting density of matter as a universal law of nature., JETP Lett. 36 (1982) 265 
[17] Y. I. Anini, The Limiting Curvature Hypothesis: Towards a Theory of Gravity Without Singularities, Current Topics in Mathematical Cosmology, Proceedings of the International Seminar held in Potsdam, Germany, 30 March - 4 April, 1998. Edited by M. Rainer and H.-J. Schmidt. World Scientific Press, 1998., p.183

[18] R. H. Brandenberger and C. Vafa, Superstrings in the Early Universe, Nucl. Phys. B 316 (1989) 391. doi:10.1016/0550-3213(89)90037-0

[19] E. Alvarez, Superstring Cosmology, Phys. Rev. D 31, 418 (1985) Erratum: [Phys. Rev. D 33, 1206 (1986)]. doi:10.1103/PhysRevD.31.418, 10.1103/PhysRevD.33.1206

[20] R. F. Baierlein, D. H. Sharp and J. A. Wheeler, Three-Dimensional Geometry as Carrier of Information about Time, Phys. Rev. 126 (1962) 1864. doi:10.1103/PhysRev.126.1864

[21] R. H. Brandenberger, V. F. Mukhanov and A. Sornborger, A Cosmological theory without singularities, Phys. Rev. D 48 (1993) 1629. doi:10.1103/PhysRevD.48.1629

[22] A. H. Chamseddine and V. Mukhanov, Nonsingular Black Hole, Eur. Phys. J. C 77 (2017) no.3, 183 doi:10.1140/epjc/s10052-017-4759-z [arXiv:1612.05861 [gr-qc]].

[23] A. H. Chamseddine and V. Mukhanov, Resolving Cosmological Singularities, JCAP 1703 (2017) no.03, 009 doi:10.1088/1475-7516/2017/03/009 [arXiv:1612.05860 [gr-qc]].

[24] S. Deser and G. W. Gibbons, Born-Infeld-Einstein actions?, Class. Quant. Grav. 15 (1998) L35 . doi:10.1088/0264-9381/15/5/001.

[25] K. S. Stelle, Renormalization of Higher Derivative Quantum Gravity, Phys. Rev. D 16 (1977) 953. doi:10.1103/PhysRevD.16.953

[26] K. S. Stelle, Classical Gravity with Higher Derivatives, Gen. Rel. Grav. 9 (1978) 353. doi:10.1007/BF00760427

[27] S. Deser and B. Tekin, Energy in generic higher curvature gravity theories, Phys. Rev. D 67 (2003) 084009 doi:10.1103/PhysRevD.67.084009 [hep-th/0212292].

[28] A. Kehagias, C. Kounnas, D. Lüst and A. Riotto, Black hole solutions in $R^{2}$ gravity, JHEP 1505 (2015) 143 doi:10.1007/JHEP05(2015)143 [arXiv:1502.04192 [hep-th]].

[29] L. Alvarez-Gaume, A. Kehagias, C. Kounnas, D. Lüst and A. Riotto, Aspects of Quadratic Gravity, Fortsch. Phys. 64 (2016) no.2-3, 176 doi:10.1002/prop.201500100 [arXiv:1505.07657 [hep-th]].

[30] S.S.Chern, Differential Geometry and Integral Geometry in Proc. Int. Congr. Math. (Edinburgth, 1985) (Cambridge Univ. Press, 1960), pp. 441-449.

[31] R.Ambartzumian, Combinatoria Integral Geometry ( John Wiley and Sons, Chichester, 1982)

[32] L.Santalo, Integral Geometry and Geometric Probability, (Addison-Wesley, Reading, Massachusetts, 1976)

[33] G. K. Savvidy and K. G. Savvidy, Interaction hierarchy: Gonihedric string and quantum gravity, Mod. Phys. Lett. A 11 (1996) 1379. doi:10.1142/S0217732396001399.

[34] J. Ambjorn, G. K. Savvidy and K. G. Savvidy, Alternative actions for quantum gravity and the intrinsic rigidity of the space-time, Nucl. Phys. B 486 (1997) 390. doi:10.1016/S0550-3213(96)00660-8.

[35] G. K. Savvidy, Quantum gravity with linear action. Intrinsic rigidity of space-time, Nucl. Phys. Proc. Suppl. 57 (1997) 104. doi:10.1016/S0920-5632(97)00358-7. 
[36] J. Ambjorn, B. Durhuus and J. Frohlich, Diseases of Triangulated Random Surface Models, and Possible Cures, Nucl. Phys. B 257 (1985) 433. doi:10.1016/0550-3213(85)90356-6

[37] R. V. Ambartsumian, G. S. Sukiasian, G. K. Savvidi and K. G. Savvidy, Alternative model of random surfaces, Phys. Lett. B 275 (1992) 99. doi:10.1016/0370-2693(92)90857-Z

[38] J. Ambjorn, J. L. Nielsen, J. Rolf and G. K. Savvidy, Spikes in quantum Regge calculus, Class. Quant. Grav. 14 (1997) 3225. doi:10.1088/0264-9381/14/12/009.

[39] G. Savvidy, The gonihedric paradigm extension of the Ising model, Mod. Phys. Lett. B 29 (2015) no.32, 1550203 doi:10.1142/S0217984915502036.

[40] E. Guendelman, A. Kaganovich, E. Nissimov and S. Pacheva, Asymptotically de Sitter and anti-de Sitter Black Holes with Confining Electric Potential, Phys. Lett. B 704 (2011) 230 doi:10.1016/j.physletb.2011.09.003,

[41] T. Regge, General Relativity Without Coordinates. , Nuovo Cim. 19 (1961) 558. doi:10.1007/BF02733251

[42] W. Pauli, Relativistic Field Theories of Elementary Particles, Rev. Mod. Phys. 13 (1941) 203. doi:10.1103/RevModPhys.13.203

[43] R. P. Feynman, The Theory of positrons, Phys. Rev. 76 (1949) 749. doi:10.1103/PhysRev.76.749

[44] R. P. Feynman, Space - time approach to quantum electrodynamics, Phys. Rev. 76 (1949) 769. doi:10.1103/PhysRev.76.769

[45] J.A.Wheeler, Regge Calculus and Schwarzschild Geometry in Relativity, Groups and Topology ed. B. DeWitt and C. DeWitt (New York: Gordon and Breach, 1964) pp 463-501

[46] M. Born and L. Infeld, Foundations of the New Field Theory, Proc. R. Soc. Lond. A 1934 (1934) 144, doi: 10.1098/rspa.1934.0059,

[47] C. Bachas, D-brane dynamics, Phys. Lett. B 374 (1996) 37 doi:10.1016/0370-2693(96)00238-9 [hep-th/9511043].

[48] H. B. Nielsen and P. Olesen, Local field theory of the dual string, Nucl. Phys. B 57 (1973) 367. doi:10.1016/0550-3213(73)90107-7 